\title{
Connection between the Wieferich congruence and divisibility of $h^{+}$
}

\author{
by
}

\author{
Stanislav Jakubec (Bratislava)
}

As is well known, the Wieferich congruence is the congruence $2^{q-1} \equiv 1$ $\left(\bmod q^{2}\right)$. Wieferich proved in 1909 that if $2^{q-1} \not \equiv 1\left(\bmod q^{2}\right)$ then for the exponent $q$ the first case of Fermat's Last Theorem holds.

The aim of this paper is to prove Theorem 1, which gives a connection between the Wieferich congruence and divisibility of $h^{+}$(the class number of the field $\left.\mathbb{Q}\left(\zeta_{p}+\zeta_{p}^{-1}\right)\right)$ by the prime $q$.

THeOREM 1. Let $q$ be an odd prime. Let $l, p$ be primes such that $p=$ $2 l+1, l \equiv 3(\bmod 4), p \equiv-1(\bmod q), p \not \equiv-1\left(\bmod q^{3}\right)$ and let the order of the prime $q$ modulo $l$ be $(l-1) / 2$. Suppose that $q$ divides $h^{+}$, the class number of the real cyclotomic field $\mathbb{Q}\left(\zeta_{p}+\zeta_{p}^{-1}\right)$. Then $2^{q-1} \equiv 1\left(\bmod q^{2}\right)$.

To prove this theorem, the following assertion from [1] will be used:

Proposition 1. Let $l, p, q$ be primes, $p \equiv 1(\bmod l), q \neq 2, q \neq l, q<p$. Let $K$ be a subfield of the field $\mathbb{Q}\left(\zeta_{p}+\zeta_{p}^{-1}\right),[K: \mathbb{Q}]=l$ and let $h_{K}$ be the class number of the field $K$. If $q \mid h_{K}$, then $q \mid N_{\mathbb{Q}\left(\zeta_{l}\right) / \mathbb{Q}}(\omega)$, where

$$
\omega=a_{1} \sum_{i \equiv 1(\bmod q)} \chi(i)+a_{2} \sum_{i \equiv 2(\bmod q)} \chi(i)+\ldots+a_{q-1} \sum_{i \equiv q-1(\bmod q)} \chi(i),
$$

and $\chi(x)$ is the Dirichlet character modulo $p, \chi(x)=\zeta_{l}^{\text {ind } x}$.

The values $a_{i}$ were calculated on the basis of the formula (4), p. 73 in [1]. Note that the numbers $a_{1}, a_{2}, \ldots, a_{q-1}$ do not depend on the prime $p$, but depend on $p$ modulo $q$. It is clear that instead of $a_{1}, \ldots, a_{q-1}$, we can consider the numbers $a a_{1}, \ldots, a a_{q-1}$ modulo $q$ for any $a \not \equiv 0(\bmod q)$.

Before we give a proof of Theorem 1 we show some connections of this paper with $q$-adic $L$-functions $L_{q}(1, \chi)$.

1991 Mathematics Subject Classification: Primary 11R29. 
The number

$$
\omega=\sum_{r=1}^{q-1} a_{r} \sum_{i \equiv r(\bmod q)} \chi(i)
$$

plays a fundamental role in the proof.

Let $F_{\gamma}^{\prime}=\left(\gamma^{q}-\gamma^{\sigma_{q}}\right) /\left(q \gamma^{\sigma_{q}}\right) \in \mathbb{Z}_{K}$ (where $\sigma_{q}$ is the Frobenius automorphism at $q$ in $K / \mathbb{Q})$, and let $\varphi: \mathbb{Z}_{K} \rightarrow \mathbb{Z}\left(\zeta_{l}\right)$ be defined by $\varphi(\alpha)=$ $c_{1}+c_{2} \zeta_{l}+\ldots+c_{l} \zeta_{l}^{l-1}$, where $\alpha=c_{1} \alpha_{1}+c_{2} \alpha_{2}+\ldots+c_{l} \alpha_{l}$ and $\alpha_{1}, \ldots, \alpha_{l}$ are Gauss periods.

It is proved in [1] that $\omega$ is equal to $\varphi\left(F_{\gamma}^{\prime}\right)$ up to a multiplicative constant $\beta \in \mathbb{Q}\left(\zeta_{l}\right)$ such that $N(\beta) \not \equiv 0(\bmod q)$.

For the $q$-adic $L$-function $L_{q}\left(1, \chi^{-1}\right)$ we have

$$
\begin{aligned}
L_{q}\left(1, \chi^{-1}\right) & =-\left(1-\frac{\chi^{-1}(q)}{q}\right) \frac{\tau\left(\chi^{-1}\right)}{p} \sum_{a=1}^{p} \chi(a) \log _{q}\left(1-\zeta_{p}^{q}\right) \\
& =u_{\chi} \frac{1}{q} \sum_{\sigma \in G} \chi(\sigma) \log _{q}\left(\gamma^{\sigma}\right),
\end{aligned}
$$

where $u_{\chi}$ is a $q$-adic unit.

The connection between $\omega$ and $L_{q}\left(1, \chi^{-1}\right)$ is stated in the following lemma.

Lemma. There is an automorphism $\sigma^{* *}$ of the field $\mathbb{Q}\left(\zeta_{l}\right)$ and a q-adic unit $v_{\chi}$ such that

$$
v_{\chi} \sigma^{* *}(\omega) \equiv L_{q}\left(1, \chi^{-1}\right)(\bmod q)
$$

Proof. Let

$$
\frac{1}{q} \log _{q} \gamma \equiv b_{1} \alpha_{1}+\ldots+b_{l} \alpha_{l}(\bmod q)
$$

Thus

$$
\begin{gathered}
\frac{1}{q} \sum_{\sigma \in G} \chi(\sigma) \log _{q}\left(\gamma^{\sigma}\right) \equiv \sum_{\sigma \in G} \chi(\sigma) \sigma\left(b_{1} \alpha_{1}+\ldots+b_{l} \alpha_{l}\right)(\bmod q), \\
\varphi\left(b_{1} \alpha_{1}+\ldots+b_{l} \alpha_{l}\right)=b_{1}+b_{2} \zeta_{l}+\ldots+b_{l} \zeta_{l}^{l-1} .
\end{gathered}
$$

By reduction of the right side of $(*)$ we deduce that there is an automorphism $\sigma^{*}$ of $\mathbb{Q}\left(\zeta_{l}\right)$ and a natural number $n$ such that

$$
\frac{1}{q} \sum_{\sigma \in G} \chi(\sigma) \log _{q}\left(\gamma^{\sigma}\right) \equiv \tau\left(\chi^{n}\right) \sigma^{*}\left(b_{1}+b_{2} \zeta_{l}+\ldots+b_{l} \zeta_{l}^{l-1}\right)(\bmod q) .
$$

It can be proved that

$$
F_{\gamma}^{\prime} \equiv \frac{1}{q} \log _{q}\left(\gamma^{\sigma_{q}}\right)(\bmod q)
$$


Finally, we have

$$
v_{\chi} \sigma^{* *}(\omega) \equiv L_{q}\left(1, \chi^{-1}\right)(\bmod q),
$$

for a suitable automorphism $\sigma^{* *}$ of $\mathbb{Q}\left(\zeta_{l}\right)$. That $v_{\chi}$ is a $q$-adic unit follows from the fact that $u_{\chi}$ and the Gauss sum $\tau\left(\chi^{n}\right)$ are both $q$-adic units.

By considering the congruence

$$
L_{q}\left(1, \chi^{-1}\right) \equiv B_{1}\left(\chi^{-1} \theta^{-1}\right)(\bmod q),
$$

(where $\theta$ is the Teichmüller character at $q$ and $B_{1}$ the generalized Bernoulli number) the result of this paper can be stated as follows:

$$
q \mid N B_{1}\left(\chi^{-1} \theta^{-1}\right) \Rightarrow \text { Wieferich congruence for prime } q \text {. }
$$

Proof of Theorem 1 . We shall prove that if $p \not \equiv-1\left(\bmod q^{3}\right)$ and $2^{q-1} \not \equiv 1\left(\bmod q^{2}\right)$ then $q$ does not divide $h^{+}$. Since the order of $q$ modulo $l$ is $(l-1) / 2$ we have $\left(\frac{q}{l}\right)=1$. From $p \equiv-1(\bmod q)$ we have $l \equiv-1$ $(\bmod q)$. Let $q \equiv 1(\bmod 4)$. Then

$$
\left(\frac{q}{l}\right)=\left(\frac{l}{q}\right)=\left(\frac{-1}{q}\right)=1 .
$$

If $q \equiv 3(\bmod 4)$, then

$$
\left(\frac{q}{l}\right)=-\left(\frac{l}{q}\right)=-\left(\frac{-1}{q}\right)=1 .
$$

As we will prove later (see Lemma 3$)$, for $p \equiv-1(\bmod q)$ we have $a_{q-1}=0$. It follows that $\omega=2 \tau$, where

$$
\tau=a_{1} \sum_{\substack{i \equiv 1(\bmod q) \\ i<p / 2}} \chi(i)+a_{2} \sum_{\substack{i \equiv 2(\bmod q) \\ i<p / 2}} \chi(i)+\ldots+a_{q-1} \sum_{\substack{i \equiv q-1(\bmod q) \\ i<p / 2}} \chi(i) .
$$

Since the order of $q$ modulo $l$ is $(l-1) / 2$, we see that $q$ splits into two divisors in $\mathbb{Q}\left(\zeta_{l}\right)$. Because $l \equiv 3(\bmod 4)$, we have $\left(\frac{-1}{l}\right)=-1$, hence if $q \mid N_{\mathbb{Q}\left(\zeta_{l}\right) / \mathbb{Q}}(\omega)$ then $q$ divides $\tau \bar{\tau}$.

The following formula holds:

$$
\tau \bar{\tau}=\sum_{\substack{i, j \equiv 1,2, \ldots, q-1(\bmod q) \\ i, j<p / 2}} a_{i} a_{j} \chi\left(i j^{-1}\right)=b_{0}+b_{1} \zeta_{l}+b_{2} \zeta_{l}^{2}+\ldots+b_{l-1} \zeta_{l}^{l-1} .
$$

Then $q \mid \tau \bar{\tau}$ if and only if $b_{0} \equiv b_{1} \equiv \ldots \equiv b_{l-1}(\bmod q)$. We shall compute the coefficient $b_{0}$.

Let $\chi\left(i j^{-1}\right)=1$. Then $i j^{-1} \equiv 1(\bmod p)$ or $i j^{-1} \equiv-1(\bmod p)$, therefore either $i-j \equiv 0(\bmod p)$ or $i+j \equiv 0(\bmod p), i, j<p / 2$. Hence $i \equiv j$ $(\bmod p)$, and therefore $i=j$. 
The following equalities hold:

$$
\begin{gathered}
\#\{i \equiv 1(\bmod q): i<p / 2\}=\frac{p+1}{2 q}, \\
\#\{i \equiv 2(\bmod q): i<p / 2\}=\frac{p+1}{2 q}, \\
\vdots \\
\#\{i \equiv q-2(\bmod q): i<p / 2\}=\frac{p+1}{2 q} .
\end{gathered}
$$

It follows that

$$
b_{0}=\frac{p+1}{2 q} \sum_{i=1}^{q-1} a_{i}^{2}
$$

Lemma 1. Let $p \equiv z(\bmod q)$. For the coefficients $a_{1}, \ldots, a_{q-1}$, the following congruence holds:

$$
a_{k} \equiv \frac{z}{2 q} \sum_{i=1}^{q-1} \frac{1}{i} \cdot\left(\overline{\frac{i-k}{z}}-\frac{\bar{i}}{z}-\frac{\overline{-k-i}}{z}+\frac{\overline{-i}}{z}\right)(\bmod q),
$$

where

$$
\overline{\frac{i-k}{z}}, \bar{i}, \overline{\frac{-k-i}{z}}, \frac{\overline{-i}}{z},
$$

are residues modulo $q$ from the interval $\langle 0, q-1\rangle$.

Proof. By the formula (4), p. 73 in [1] we have

$$
\frac{\left(\zeta_{p}-1\right)^{q}-\left(\zeta_{p}^{q}-1\right)}{q} \sum_{i=1}^{p-1} i \zeta_{p}^{q i}=c_{0}+c_{1} \zeta_{p}+c_{2} \zeta_{p}^{2}+\ldots+c_{p-1} \zeta_{p}^{p-1}
$$

Let $\zeta_{p}^{i} \zeta_{p}^{q j}=1$. Then $i+q j \equiv 0(\bmod p)$, and therefore

$$
j \equiv \frac{-i}{q}(\bmod p), \quad 0 \leq j<p .
$$

Hence

$$
c_{0} \equiv \frac{1}{q} \sum_{i=1}^{q-1} \frac{\overline{-i}}{q}\left(\begin{array}{c}
q \\
q-i
\end{array}\right)(-1)^{q-i}(\bmod q)
$$

where $\frac{\overline{\frac{-i}{q}}}{q}$ is a residue modulo $p$, with $0 \leq \overline{\overline{\frac{a i}{q}}}<p$. According to [1],

$$
a_{k}=c_{k}-c_{0}=\frac{1}{q} \sum_{i=1}^{q-1}\left(\begin{array}{c}
q \\
q-i
\end{array}\right)(-1)^{q-i}\left(\overline{\frac{k-i}{q}}-\frac{\overline{-i}}{q}\right) .
$$


Replacing $i$ by $q-i$ and using $\left(\begin{array}{l}q \\ i\end{array}\right)=\left(\begin{array}{c}q \\ q-i\end{array}\right)$ we get

$$
a_{k} \equiv \frac{1}{2} \frac{z}{q} \sum_{i=1}^{q-1}\left(\begin{array}{l}
q \\
i
\end{array}\right)(-1)^{i+1}\left(\frac{\overline{k-i}}{q}-\frac{\overline{-i}}{q}-\frac{\overline{k+i}}{q}+\frac{\bar{i}}{q}\right)(\bmod q) .
$$

Let $p=a q+z$. Let $x_{1}$ be such that

$$
x_{1} p+k-i \equiv 0(\bmod q), \quad 0 \leq x_{1}<q .
$$

The numbers $x_{2}, x_{3}, x_{4}$ will be defined analogously. Then

$$
\begin{aligned}
\frac{\overline{k-i}}{q} & =\frac{x_{1}(a q+z)+k-i}{q}=a x_{1}+\frac{x_{1} z+k-i}{q}, \\
\frac{\overline{-i}}{q} & =\frac{x_{2}(a q+z)-i}{q}=a x_{2}+\frac{x_{2} z-i}{q}, \\
\frac{\overline{k+i}}{q} & =\frac{x_{3}(a q+z)+k+i}{q}=a x_{3}+\frac{x_{3} z+k+i}{q}, \\
\frac{\bar{i}}{q} & =\frac{x_{4}(a q+z)+i}{q}=a x_{4}+\frac{x_{4} z+i}{q} .
\end{aligned}
$$

Hence

$$
\begin{aligned}
& \frac{\overline{k-i}}{q}-\frac{\overline{-i}}{q}-\frac{\overline{k+i}}{q}+\frac{\bar{i}}{q} \\
& =a\left(x_{1}-x_{2}-x_{3}+x_{4}\right)+\frac{x_{1} z+k-i}{q}-\frac{x_{2} z-i}{q}-\frac{x_{3} z+k+i}{q}+\frac{x_{4} z+i}{q} .
\end{aligned}
$$

It is easy to see that

$$
x_{1}-x_{2}-x_{3}+x_{4} \equiv 0(\bmod q) .
$$

The assertion of Lemma 1 now follows from the congruence

$$
\frac{1}{q}\left(\begin{array}{l}
q \\
i
\end{array}\right)(-1)^{i+1} \equiv \frac{1}{i}(\bmod q)
$$

Lemma 2. Let $p \equiv z(\bmod q), 0<z<q$. Then $a_{k} \equiv a_{z-k}(\bmod q)$.

Proof. This follows from Lemma 1.

Let $r<l$. Then $g^{r} \equiv 2$ or $-2(\bmod p)$. We shall compute the coefficient $b_{r}$.

Let $\chi\left(i j^{-1}\right)=\zeta_{l}^{r}$. Then either $\operatorname{ind}\left(i j^{-1}\right)=r$ or $\operatorname{ind}\left(i j^{-1}\right)=r+l$ and therefore either $i j^{-1} \equiv 2(\bmod p)$ or $i j^{-1} \equiv-2(\bmod p), i, j<p / 2$. Hence by Lemma 2 we have

$$
b_{r} \equiv \frac{p+1}{2 q} \sum_{i=1}^{q-1} a_{i} a_{2 i}(\bmod q) .
$$


Therefore if $q \mid \tau \bar{\tau}$, then

If

$$
\frac{p+1}{2 q}\left(\sum_{i=1}^{q-1} a_{i} a_{2 i}-\sum_{i=1}^{q-1} a_{i}^{2}\right) \equiv 0(\bmod q) .
$$

then

$$
\sum_{i=1}^{q-1} a_{i} a_{2 i}-\sum_{i=1}^{q-1} a_{i}^{2} \not \equiv 0(\bmod q)
$$

and hence $p \equiv-1\left(\bmod q^{2}\right)$.

$$
\frac{p+1}{2 q} \equiv 0(\bmod q),
$$

We shall prove that

$$
\sum_{i=1}^{q-1} a_{i} a_{2 i}-\sum_{i=1}^{q-1} a_{i}^{2} \equiv-\frac{2^{q-1}-1}{q}(\bmod q) .
$$

Lemma 3. Let $p \equiv-1(\bmod q)$. Then

$$
a_{k} \equiv \sum_{i=1}^{k} \frac{1}{i}(\bmod q) \quad \text { for } k=1,2, \ldots, q-1 .
$$

Proof. It is easy to see that

$$
\begin{aligned}
\frac{\overline{i-k}}{q-1}=i-k+\delta_{i, k}, \quad \text { where } \delta_{i, k}= \begin{cases}0, & k \leq i, \\
q, & i<k,\end{cases}
\end{aligned}
$$

It follows that

$$
a_{k} \equiv \frac{1}{q} \sum_{i=1}^{q-1} \frac{1}{i}\left(\delta_{i, k}+\beta_{i, k}-q\right)(\bmod q) .
$$

Analysing all cases we get the congruence of Lemma 3.

LEMMA 4. The following congruence holds:

$$
\sum_{i=1}^{q-1} a_{i}^{2} \equiv-2(\bmod q) .
$$


Proof. It is easy to see that

$$
\begin{aligned}
\sum_{i=1}^{q-1} a_{i}^{2} & \\
\equiv & (q-1) 1^{2}+(q-2) \frac{1}{2^{2}}+(q-3) \frac{1}{3^{2}}+\ldots+1 \cdot \frac{1}{(q-1)^{2}} \\
& +2\left(1 \cdot(-1)(q-2)+\frac{1}{2}(-1)(q-3)+\ldots+\frac{1}{q-2}(-1)(q-(q-1))\right) \\
\equiv & -\left(1+\frac{1}{2}+\ldots+\frac{1}{q-1}\right)+2\left(\frac{2}{1}+\frac{3}{2}+\ldots+\frac{q-1}{q-2}\right) \\
\equiv & -2(\bmod q) .
\end{aligned}
$$

Lemma 5. Let $m=(q-1) / 2$. The following congruence holds:

$$
\sum_{i=1}^{q-1} a_{i} a_{2 i} \equiv\left(-1+\frac{1}{2}-\frac{1}{3}+\ldots+(-1)^{m} \frac{1}{m}\right)-2(\bmod q) .
$$

Proof. Let $m \equiv 0(\bmod 2)$. It is easy to see that

$$
\begin{aligned}
\sum_{i=1}^{q-1} a_{i} a_{2 i} \equiv & 1\left(1+\frac{1}{2}\right)+\left(1+\frac{1}{2}\right)\left(1+\frac{1}{2}+\frac{1}{3}+\frac{1}{4}\right) \\
& +\ldots+\left(1+\frac{1}{2}+\ldots+\frac{1}{m}\right)\left(1+\frac{1}{2}+\ldots+\frac{1}{2 m}\right) \\
& +\left(1+\frac{1}{2}+\ldots+\frac{1}{m-1}\right)\left(1+\frac{1}{2}+\ldots+\frac{1}{2 m-1}\right) \\
& +\left(1+\frac{1}{2}+\ldots+\frac{1}{m-2}\right)\left(1+\frac{1}{2}+\ldots+\frac{1}{2 m-3}\right) \\
& +\ldots+1 \cdot\left(1+\frac{1}{2}+\frac{1}{3}\right) .
\end{aligned}
$$

Multiplying out from the left by the numbers $1, \frac{1}{2}, \frac{1}{3}, \ldots, \frac{1}{m}$, one after another, we have

$$
\begin{aligned}
& \sum_{i=1}^{q-1} a_{i} a_{2 i} \\
& \equiv 1 \cdot\left((q-2)+(q-2) \frac{1}{2}+(q-3) \frac{1}{3}+\ldots+1 \cdot \frac{1}{q-1}\right) \\
& \quad+\frac{1}{2}\left((q-4)\left(1+\frac{1}{2}+\frac{1}{3}\right)+(q-4) \frac{1}{4}+(q-5) \frac{1}{5}+\ldots+1 \cdot \frac{1}{q-1}\right)
\end{aligned}
$$




$$
\begin{aligned}
& +\frac{1}{3}\left((q-6)\left(1+\frac{1}{2}+\ldots+\frac{1}{5}\right)+(q-6) \frac{1}{6}+(q-7) \frac{1}{7}+\ldots+1 \cdot \frac{1}{q-1}\right) \\
& +\ldots+\frac{1}{i}\left((q-2 i)\left(1+\frac{1}{2}+\ldots+\frac{1}{2 i-1}\right)\right. \\
& \left.+(q-2 i) \frac{1}{2 i}+\ldots+1 \cdot \frac{1}{q-1}\right) \\
& +\ldots+\frac{1}{m}\left((q-2 m)\left(1+\frac{1}{2}+\ldots+\frac{1}{2 m-1}\right)+\frac{1}{q-1}\right)(\bmod q) .
\end{aligned}
$$

It follows that

$$
\begin{aligned}
\sum_{i=1}^{q-1} a_{i} a_{2 i} \equiv & -2-2\left(1+\frac{1}{2}+\frac{1}{3}\right)-2\left(1+\frac{1}{2}+\ldots+\frac{1}{5}\right) \\
& -\ldots-2\left(1+\frac{1}{2}+\ldots+\frac{1}{2 m-1}\right)+q-1(\bmod q) .
\end{aligned}
$$

Hence

$$
\begin{aligned}
\sum_{i=1}^{q-1} a_{i} a_{2 i} \equiv & -2 m-2(m-1)\left(\frac{1}{2}+\frac{1}{3}\right)-2(m-2)\left(\frac{1}{4}+\frac{1}{5}\right) \\
& -\ldots-2\left(\frac{1}{2 m-2}+\frac{1}{2 m-1}\right)-1(\bmod q) .
\end{aligned}
$$

From this we obtain

$$
\begin{aligned}
\sum_{i=1}^{q-1} a_{i} a_{2 i} \equiv & -2\left(m\left(1+\frac{1}{2}+\ldots+\frac{1}{m-1}\right)\right. \\
& \left.-\left(m-\left(\frac{1}{3}+\frac{1}{5}+\ldots+\frac{1}{m+1}\right)\right)\right)-1(\bmod q) .
\end{aligned}
$$

Therefore, we have

$$
\sum_{i=1}^{q-1} a_{i} a_{2 i} \equiv\left(-1+\frac{1}{2}-\frac{1}{3}+\ldots+(-1)^{m} \frac{1}{m}\right)-2(\bmod q) .
$$

In the case $m \equiv 1(\bmod 2)$, we proceed analogously.

The following congruence is known:

$$
1-\frac{1}{2}+\frac{1}{3}+\ldots+(-1)^{m+1} \frac{1}{m} \equiv \frac{2^{q-1}-1}{q}(\bmod q) .
$$

This easily implies that if $q \mid h^{+}$and $2^{q-1} \not \equiv 1\left(\bmod q^{2}\right)$ then

$$
p \equiv-1\left(\bmod q^{2}\right) \text {. }
$$


Lemma 6. If $q \mid h^{+}$and $p \equiv-1\left(\bmod q^{2}\right)$, then $p \equiv-1\left(\bmod q^{3}\right)$.

Proof. Let $s<l$. Then $g^{s} \equiv 2 q$ or $-2 q(\bmod p)$. We shall compute the coefficient $b_{s}$.

Let $\chi\left(i j^{-1}\right)=\zeta_{l}^{s}$. Then either $\operatorname{ind}\left(i j^{-1}\right)=s$ or ind $\left(i j^{-1}\right)=s+l$ and therefore either $i j^{-1} \equiv 2 q(\bmod p)$ or $i j^{-1} \equiv-2 q(\bmod p) i, j<p / 2$.

Consider the intervals

$$
\left(0, \frac{p}{2 q}\right),\left(\frac{p}{2 q}, \frac{2 p}{2 q}\right),\left(\frac{2 p}{2 q}, \frac{3 p}{2 q}\right), \ldots,\left(\frac{(q-1) p}{2 q}, \frac{q p}{2 q}\right) .
$$

If

$$
x \in\left(\frac{i p}{2 q}, \frac{(i+1) p}{2 q}\right),
$$

then ip $<2 q x<(i+1) p$. Reducing modulo $p$ we get

$$
2 q x-i p \equiv-i(q-1) \equiv i(\bmod q) .
$$

Let $p=a q^{2}-1$. Then

$$
\#\left\{x \in\left(\frac{i p}{2 q}, \frac{(i+1) p}{2 q}\right), x \equiv k(\bmod q)\right\}=\frac{a}{2}
$$

for $k=1,2, \ldots, q-1, i=1,2, \ldots, q-1$. By Lemma 2 , it follows that

$$
\begin{aligned}
b_{s} \equiv & \frac{a}{2} a_{1}\left(a_{1}+a_{2}+\ldots+a_{q-1}\right)+\frac{a}{2} a_{2}\left(a_{1}+a_{2}+\ldots+a_{q-1}\right) \\
& +\ldots+\frac{a}{2} a_{q-1}\left(a_{1}+a_{2}+\ldots+a_{q-1}\right) .
\end{aligned}
$$

Since

$$
a_{1}+a_{2}+\ldots+a_{q-1} \equiv 1(\bmod q),
$$

we have $b_{s} \equiv \frac{a}{2}(\bmod q)$. If $q \mid h^{+}$then $b_{s} \equiv b_{0} \equiv 0(\bmod q)$ and hence $a \equiv 0(\bmod q)$. It follows that

$$
p \equiv-1\left(\bmod q^{3}\right) .
$$

Theorem 1 is proved.

Remark. For $q<6 \cdot 10^{9}$ there are exactly two primes satisfying the congruence $2^{q-1} \equiv 1\left(\bmod q^{2}\right)$, namely $q=1093$ and $q=3511$. Hence Theorem 1 does not give any information on divisibility of $h^{+}$for these two primes.

This default can be removed in the following way. Consider the coefficient $b_{t}$ corresponding to the congruences

$$
i j^{-1} \equiv 3(\bmod p) \quad \text { or } \quad i j^{-1} \equiv-3(\bmod p), \quad i, j<p / 2 .
$$

Then

$$
b_{t} \equiv \frac{p+1}{3 q} \sum_{i=1}^{q-1} a_{i} a_{3 i}+\frac{p+1}{6 q} \sum_{i=1}^{q-1} a_{i} a_{3 i+1}(\bmod q) .
$$


Hence it is enough to prove that

(**) $\quad \frac{p+1}{3 q} \sum_{i=1}^{q-1} a_{i} a_{3 i}+\frac{p+1}{6 q} \sum_{i=1}^{q-1} a_{i} a_{3 i+1}-\frac{p+1}{2 q} \sum_{i=1}^{q-1} a_{i}^{2} \not \equiv 0(\bmod q)$.

By a numerical calculation for $q=1093$ and $q=3511$ we find that (**) holds. Therefore if $p \not \equiv-1\left(\bmod q^{3}\right)$ for $q=1093$ and $q=3511$, then under the assumptions of Theorem 1, neither 1093 nor 3511 divides $h^{+}$.

Acknowledgements. I am grateful to the referee for his suggestions concerning $q$-adic $L$-functions.

\section{References}

[1] S. Jakubec, On divisibility of class number of real Abelian fields of prime conductor, Abh. Math. Sem. Univ. Hamburg 63 (1993), 67-86.

MATEMATICKÝ ÚSTAV SAV

ŠTEFÁNIKOVA 49

81473 BRATISLAVA, SLOVAKIA

Received on 24.2.1994

and in revised form on 21.9.1994 